Ciencia, Ambiente y Clima, Vol. 2, No. 1, enero-junio, 2019 • ISSN (impreso): 2636-2317 • ISSN (en línea): 2636-2333

DOI: https://doi.org/10.22206/cac.2019.v2i1.pp49-50

\title{
NOTA DEL XI SEMINARIO DE INVESTIGACIÓN CIENTÍFICA E INNOVACIÓN TECNOLÓGICA
}

\section{Note from the XI Seminar on Scientific Research and Technological Innovation}

\author{
Alfredo Dalmau Disla \\ Universidad Autónoma de Santo Domingo, Escuela de Biología, Facultad de Ciencias, República Dominicana \\ Correo-e: dalmaudislaa@gmail.com
}

Recibido: 25/3/2019 • Aprobado: 4/4/2019

Cómo citar: Dalmau Disla, A. (2019). Nota del XI Seminario de Investigación Científica e Innovación Tecnológica. Ciencia, Ambiente y Clima, 2(1), 49-50. doi: https://doi.org/10.22206/cac.2019.v2i1.pp49-50

El décimo primer el Seminario de Investigación Científica e Innovación Tecnológica fue organizado por el Ministerio de Educación Superior Ciencia y Tecnología (MESCyT) y se celebró durante los días 7, 8 y 9 de marzo 2018, en el Hotel Sheraton de la ciudad de Santo Domingo.

Este encuentro fue el espacio en el que se presentaron unas 82 ponencias de diferentes temas de investigación, realizadas por estudiantes e investigadores de diferentes áreas científicas. Participaron 23 instituciones del ámbito educativo y científico: Pontificia Universidad Católica Madre y Maestra (PUCMM), Universidad Autónoma de Santo Domingo (UASD), Universidad Nacional Pedro Henríquez Ureña (UNPHU), Centro de Educación Médica de Amistad Dominico Japonesa (CEMADOJA), Universidad Iberoamericana (UNIBE), Servicio Geológico Nacional (SGN), Universidad Tecnológica de Santiago
(UTESA), Instituto Dominicano de Investigaciones Agropecuarias y Forestales (IDIAF), Universidad Agroforestal Fernando Arturo de Meriño (UAFAM), Universidad ISA (ISA), Universidad Tecnológica del Cibao Oriental (UTECO), Korea Digital Hospital Export Agency (KOHEA), Parque Cibernético Santo Domingo (PCSD), Universidad Nacional Evangélica (UNEV), Universidad APEC (UNAPEC), Servicio Europeo de Acción Exterior (EEAS), Centro de Investigación Biomédicas y Clínicas-Hospital Regional Universitario José María Cabral y Báez (CINBIOCLI), Instituto de Innovación en Biotecnología e Industria (IIBI), Instituto Nacional de Recursos Hidráulicos (INDRHI), Universidad Abierta para Adultos (UAPA), Centro Nacional de Investigaciones en Salud Materno Infantil Dr. Hugo Mendoza (CENISMI), Instituto Tecnológico de las Américas (ITLA), Federation of Korean Industries (FKI). 
El objetivo principal del XI Seminario de Investigación Cientifica e Innovación Tecnológica fue divulgar entre la comunidad científica los resultados de investigación de los años 2016-2017 del Fondo Nacional de Innovación y Desarrollo Científico y Tecnológico (FONDOCYT).
La realización de estos seminarios por el Ministerio de Educación Superior, Ciencia y Tecnología (MESCyT), constituye una excelente alternativa y una oportunidad para el encuentro y conversación científica en el desarrollo de la ciencia en República Dominicana. 